\title{
Spatial Identification of Defect Sites in Individual Carbon Nanotubes Using Micro-Raman Spectroscopy
}

\author{
D. McClain*, W. Hudson*, T. Ngyuen*, J. Jiao*, K. O’Brien** \\ * Department of Physics, Portland State University, Portland, OR 97207-0751 \\ ** Components Research, Intel Corporation, Hillsboro, OR 97124
}

The role of defects in the structural and electrical properties of carbon nanotubes (CNTs) is becoming increasingly important for a number of applications. These defects have been traced to changes in transport behavior (e.g. from metallic to semiconducting) in transistors [1], compressive buckling in thin films [2], and electrochemical sensitivity in sensors [3]. Raman spectroscopy has been successfully used for determining CNT defect density and chirality of bulk samples but it has only recently been applied to individual tubes [4]. The advantages of this technique include its speed, non-destructive nature and ability to characterize disorder in $\mathrm{sp}^{2}$ carbon materials like CNTs. The disorder-induced D-bands are the principle Raman signal used to determine the presence of defects in nanotubes [5]. However, use of these peaks to map the locations of defects in CNTs is complicated by very weak D-band intensities in single, isolated tubes.

We have begun to address this shortcoming by developing a technique that incorporates highresolution SEM imaging, oversampled micro-Raman spectroscopy and graphical analysis techniques to locate sites of increased defect density in individual CNTs with high spatial resolution. The CNTs used in this report, grown from isolated Fe catalyst islands, have been identified by TEM as singlewalled [Figure 1(a)]. High-resolution SEM micrographs of each of these islands are obtained using low-voltage contrast imaging to visualize the overall morphology of the SWCNTs and establish the size of the required Raman scan area. The Raman spectra of the as-grown tubes are then obtained using a Horiba LabRAM HR confocal spectrometer with a 532nm excitation laser. The scan parameters including beam intensity, step size and sampling time are carefully calibrated to increase the clarity of the D-band [Figure 1(b)]. The spectra from each point in the scan area are then assembled into a 2D Raman intensity map of the CNTs. Since the position of the D-band itself varies within a narrow window $\left(\Delta \omega_{\mathrm{D}}=24 \mathrm{~cm}^{-1}\right)$, the resolution of the map is optimized through a Matlab program that allows us to quickly identify the specific D-band wavenumber that yields the highest resolution across the length of the tube [Figure 1(c)].

The final step in the process involves overlaying the D-band intensity map with the SEM image of the CNTs as shown in Figure 2. This creates an immediate, highly visual match between prominent areas of CNT morphology such as kinks or bends in the SEM image and areas of increased disorder as indicated by the D-band intensity map. For example, the areas of peak D-band intensity in Figure 2 correspond directly to those locations in the SEM micrograph where the CNTs intersect or wrap around one another. These points are where the walls of the tubes are under the most stress and consequently where the graphene lattice is most likely to exhibit stress-induced defects such as vacancies and Stone-Wales [2]. Evidence of these defects is provided by sharp D-band peaks (increased disorder) at those locations, particularly on the left side of Figure 2. These results demonstrate a significant advance for defect mapping in carbon nanotubes with complex morphologies and confirms the promise of this technique. 

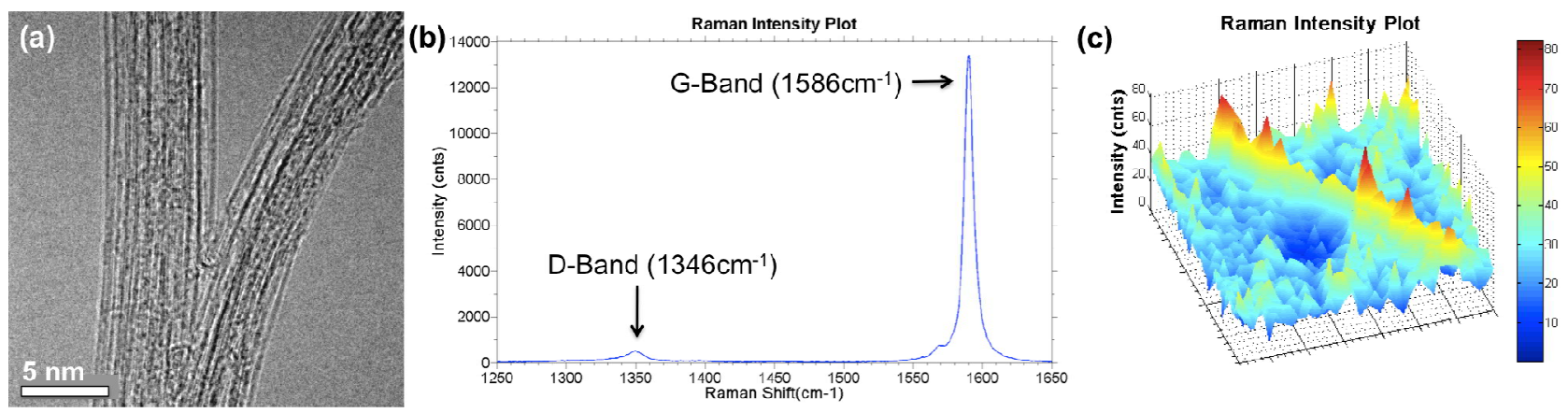

FIG. 1. (a) TEM image of as-grown SWCNTs using identical growth recipe to those used in this study. (b) The relative G- and D-band intensities of a single isolated SWCNT. The intensity of the D-band is associated with the number of defects in the tube. (c) The Matlab program created to select the correct D-band wavenumber for increased resolution in the defect map.

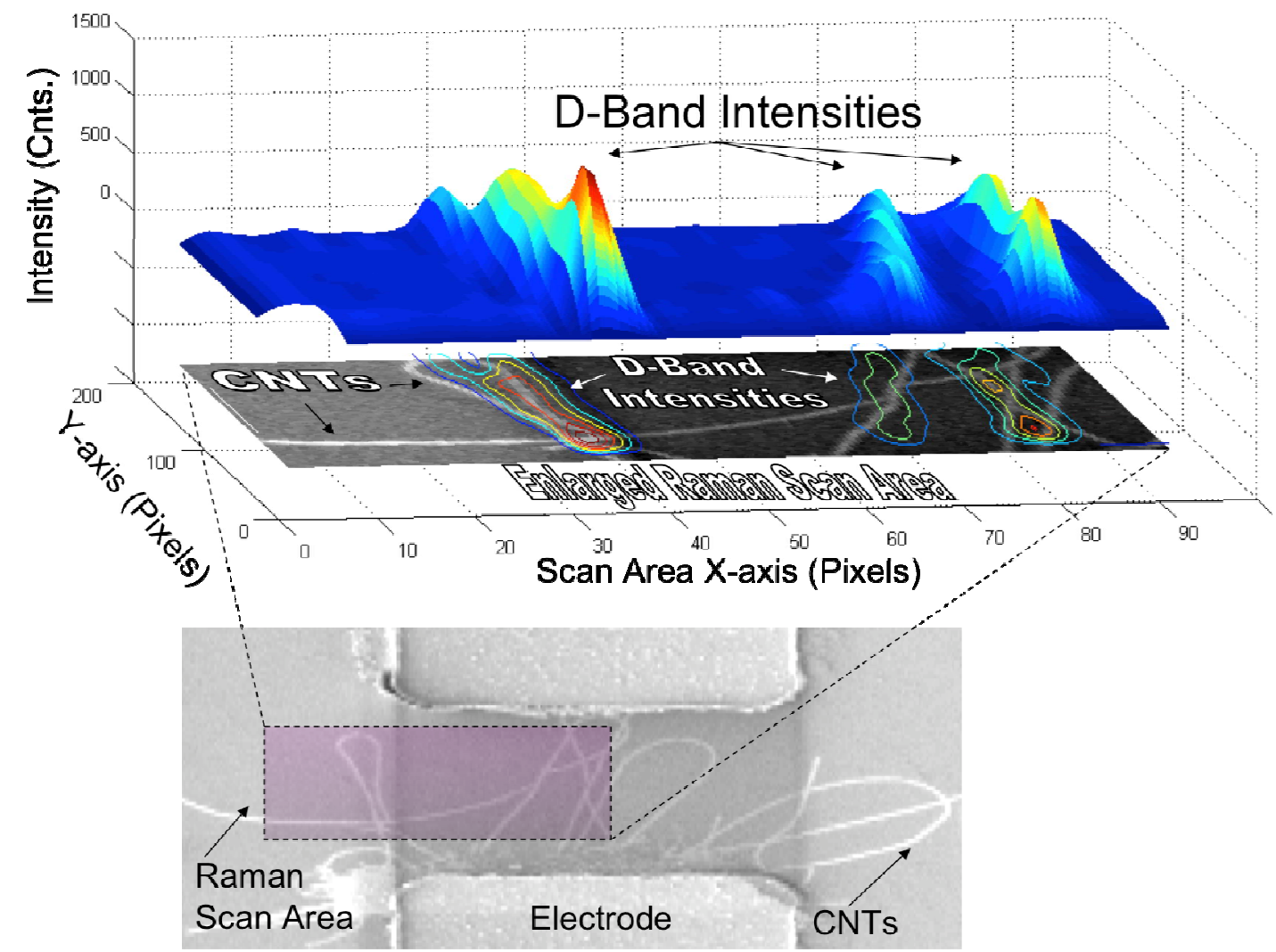

FIG. 2. Lower image: Low-voltage contrast SEM micrograph of as-grown SWCNTs with micro-Raman mapping area indicated by the shaded box. Upper Image: D-band intensity map overlaid onto the corresponding locations in the SEM micrograph. Note the strong correlation between the highest D-band peak (indicating increased CNT disorder) and the point where several CNTs meet and wrap around one another.

\section{References}

[1] Y. Fan et al., Nature Mat. 4 (2005) 906.

[2] X. Hao et al., Comp. Sci. Tech. 68 (2008) 1809.

[3] J.A. Robinson et al., Nano Lett. 6 (2006) 1747.

[4] M.S. Dresselhaus et al., Nano Lett. (2010) DOI: 10.1021/nl904286r

[5] M.S. Dresselhaus et al., Phys Rep. (2004) DOI:10.1016/j.physrep.2004.10.006 